\title{
Die Situationslogik ethnischer Konflikte
} Auch eine Anmerkung zum Beitrag „Ethnische Mobilisierung und die
Logik von Identitätskämpfen“ von Klaus Eder und Oliver Schmidtke
(ZfS 6/98)

\section{Hartmut Esser}

Universität Mannheim, Fakultät für Sozialwissenschaften, Seminargebäude A5, D-68131 Mannheim

Zusammenfassung: Der Beitrag behandelt die verschiedenen Aspekte der „Situationslogik“, aus der heraus ethnische Konflikte entstehen und eine bestimmte Eigendynamik auch gegen die Absichten der involvierten Akteure entwickeln. Dabei spielt das soziale Framing der Situation als die wechselseitige Konstitution einer bestimmten ethnischen „Definition“ der Situation eine zentrale Rolle. Das theoretische Mittel der Modellierung ist das Konzept der soziologischen Erklärung, einschließlich der Nutzung einer „kognitivistischen“ Variante der sog. Wert-Erwartungstheorie. In einem zweiten Teil werden die Verbindungen und Übereinstimmungen dieses Ansatzes mit dem Konzept der Situationslogik nach Karl R. Popper gezeigt, speziell in Abgrenzung vom sog. Psychologismus. Den Abschluß bildet eine Entgegnung auf den Beitrag von Eder und Schmidtke (Band 27, 1998, S. 418-437 der ZfS), in dem der vorgestellte Ansatz als psychologistisch kritisiert wurde und in dem als Alternative das Konzept des "Situationalismus" vorgeschlagen worden war.

Vor etwa 20 Jahren begann, aus der Fehlentwicklung des sog. verhaltenstheoretischen Ansatzes hervorgehend und dessen "reduktionistische“ Schwächen korrigierend, der sog. Rational-Choice-Ansatz in der Soziologie Fuß zu fassen. Inzwischen gibt es RC-Erklärungen zunehmend für Phänomene, die sich, zumindest vordergründig, durch eine besondere „Irrationalität" auszeichnen, wie Altruismus, Diskriminierungen, Religiösität oder die ,wertrationale“ Befolgung von Normen. Schon seit einiger Zeit gibt es RC-Erklärungen auch für die Entstehung von ethnischen Differenzierungen, für die Mobilisierung von ethnischen Konflikten und für die Emergenz und die Stabilität interethnischer Kooperation. ${ }^{1}$ Es sind, wenn man den neueren Übersichten zu dem Thema glauben darf, die einzigen Ansätze zu einer theoretisch-erklärenden Befassung mit dem Thema (vgl. Brubaker/Laitin 1998).

Ethnische Konflikte, speziell solche, die mit blutiger Gewalt ausgetragen werden, bilden ohne Zweifel eine ganz besondere Herausforderung für einen Ansatz, der u.a. von einer zwar beschränkten, aber einer immer auch an den „objektiven" situationalen Verhältnissen orientierten "Vernunft" der Menschen als universaler anthropologischer Konstante ausgeht und die Entwicklung einer allge-

\footnotetext{
${ }^{1}$ Vgl. dazu u.a. Axelrod 1987: 132 ff.; Banton 1967, 1993, 1994; Brubaker/Laitin 1998: 437-441; Esser 1980, 1985, 1988, 1996a, 1996b, 1997; Fearon/Laitin 1996; Gartner/Segura 1997; Hardin 1995; Hechter 1995; Laitin 1995; Ludemann 1998; Weingast 1998. Vgl. auch die Verweise auf den Ansatz bei Eder/Schmidtke 1998: $419 \mathrm{ff}$.
}

meinen Sozialtheorie im Auge hat. Wenn die Erklärung dieser Vorgänge mit den Mitteln des RC-Ansatzes gelänge, dann wäre das schon ein starker Beleg für die - von der herkömmlichen Soziologie nach wie vor bestrittene - universelle Brauchbarkeit der RC-Theorie.

Daher verwundert es nicht, daß nun auch die Versuche zur RC-Erklärung ethnischer Konflikte zum Anlaß werden, die grundsätzliche Brauchbarkeit des RC-Ansatzes zu bestreiten, wie das Eder und Schmidtke in ihrem Beitrag tun (Eder/ Schmidtke 1998). Die folgenden Darlegungen sind daher auch eine Entgegnung auf die wiederholt und in sehr verschiedenen Zusammenhängen vorgebrachten Behauptungen grundsätzlicher Defizite des RC-Ansatzes (vgl. etwa neuerdings noch Mozetic 1998), insbesondere bei der Erklärung von Prozessen der symbolisch-interaktiven Konstitution und Konstruktion von kollektiven Identitäten, Identifikationen und Frames, von denen die Konstruktion von Ethnizität ja nur ein Spezialfall ist.

Dazu wird zunächst das „RC+-Modell“ der Erklärung ethnischer Konflikte, das Eder und Schmidtke aus verstreuten Veröffentlichungen zusammenzufügen versucht haben, einmal im Zusammenhang dargestellt, weil sich schon daraus die meisten Einwände entkräften lassen. Zweitens werden die, eigentlich auf der Hand liegenden, aber offenbar nicht überall bekannten, Beziehungen des Konzepts der soziologischen Erklärung, das dem $\mathrm{RC}(+)$ Modell der Erklärung ethnischer Konflikte zugrunde liegt, zu dem Konzept der Situationslogik von Karl $\mathbf{R}$. Popper und zu den Unterschieden zum „Psychologismus“ verdeutlicht, an den Eder und Schmidtke wohl mit ihren 
Einwänden gegen den $\mathbf{R C}(+)$-Ansatz gedacht haben. Und schließlich werden, daran anschließend, die speziellen Einwände und „alternativen“ Vorschläge von Eder und Schmidtke besprochen.

Die Ausführungen dienen, vor allem in den methodologischen Teilen, der Klarstellung einiger Einzelheiten, aus deren Fehlwahrnehmung sich erst der größte Teil der kritischen Einwände gegen den $\mathbf{R C}(+)$-Ansatz, nicht nur bei Eder und Schmidtke, ergibt.

\section{Die RC-Erklärung ethnischer Konflikte}

Die RC-Erklärung der Entstehung und des $\mathrm{Ab}$ laufs ethnischer Konflikte unterscheidet - in Einklang mit den wichtigsten speziellen Beiträgen zu dem Problembereich der "ethnischen“ Konflikte (vgl. etwa Horowitz 1985; Ganter 1995; Wimmer 1995) und mit den herkömmlichen soziologischen Konzeptualisierungen der Entstehung von Konflikten und sozialen Bewegungen (vgl. etwa Giegel 1998: 16 ff.; Rucht 1994: Abschnitt 1.2) - analytisch vier verschiedene grundlegende Konstellationen und Problemfelder. Diese müssen in den „empirischen" Vorgängen nicht alle vorkommen, sind aber als typische Probleme stets vorhanden: Der strukturelle Hintergrund des Konflikts, das ethnische Framing des Konflikts, das damit eng verbundene Problem der Mobilisierung der ethnischen „Bewegung" und die schließliche ,situationslogische" Eigendynamik des Konfliktes und der damit verbundenen kulturellen Codierungen. Es werden also nicht grundlegend neue empirische Phänomene beachtet, jedoch versucht, den Vorgängen eine explizit erklärende Grundlage zu geben und dadurch die "Situationslogik" des Geschehens mit der nötigen „Logik“ zu versehen, die sie über die verbalen Erzählungen hinaus immer benötigt, damit von einer Situationslogik überhaupt die Rede sein kann.

Das folgende idealtypische und daher zwingend vereinfachende Modell orientiert sich nicht an einzelnen empirischen Vorgängen, sondern versucht aus der Synopse ganz unterschiedlicher Fälle und theoretischer Beiträge zu deren Erklärung eine analytische Abstraktion der wichtigsten Stadien des Ablaufs ethnischer Konflikte und der mit ihrer Erklärung auftretenden Problemfelder. ${ }^{2}$ Die Grund-

\footnotetext{
${ }^{2}$ Für die Analyse einiger konkreter ethnischer Konflikte aus der expliziten Sicht des RC-Ansatzes vgl. u.a. Hardin (1995, Kapitel 6) für - das ehemalige - Jugoslawien, für Nordirland, Somalia, Burundi und Rwanda und Kanada; oder Weingast (1998) für - das ehemalige - Jugoslawien und den amerikanischen Bürgerkrieg. Für die strukturell-
}

hypothese ist, daß ethnische Konflikte einen Spezialfall des Phänomens sozialer Bewegungen allgemein darstellen und daß einige Erklärungsargumente dafür auf die ethnischen Konflikte angewandt werden können. Die Besonderheit der „ethnischen“ Konflikte besteht darin, daß die Vorgänge hier unter einer ,ethnischen" Rahmung stattfinden. Sie sind damit ihrerseits ein Spezialfall von kollektiven Konflikten, die unter wertrationalen Codierungen gerahmt sind, wie etwa religiöse, rassische oder regionale Konflikte. Auf unterschiedliche Formen ethnischer Konflikte - wie Autonomiebestrebungen, Separatismus, Rassenunruhen, soziale Distanzen, fremdenfeindliche Gewalt oder Pogrome - wird, obwohl sich dieses anbieten würde, aus Platzgründen nicht näher eingegangen, weil das grundlegende Modell - mit gewissen Modifikationen natürlich - auf alle diese Fälle anwendbar ist.

Wir gehen die vier Aspekte der Reihe nach durch.

\section{Der strukturelle Hintergrund: Der Kampf um die Bewertung des spezifischen Kapitals}

Der Ausgangspunkt der RC-Erklärung der Entstehung ethnischer Konflikte ist eine strukturelle und daher objektive Annahme: Es gibt einen, stets mit auch existentiellen Interessen verbundenen sog. Konstant- bzw. Nullsummenkonflikt, bei dem Gewinne der einen Seite nur auf Kosten der jeweiligen Gegenseite möglich sind. Bei ethnischen Konflikten geht es im Kern um die Durchsetzung einer grundlegenden „Verfassung“ für die jeweilige Gesellschaft und damit um die Etablierung bestimmter grundlegender Regeln der Bewertung bestimmter Lebensweisen und dem damit verbundenen kulturellem $\mathrm{Ka}$ pital. Daher steht dabei typischerweise die $\mathrm{Ab}$-oder gar Umwertung eines für die ethnischen Gruppen sehr spezifischen Kapitals auf dem Spiel. „Spezifisch" ist das Kapital einer ethnischen Gruppe in dem $\mathrm{Maße}$, wie sein Wert ausschließlich an die anerkannte und mit Karrieren verbundene politische und soziale Existenz der jeweiligen ethnischen Gruppe gebunden ist. Und brisant wird der Kampf um die Verfassung dann insbesondere dadurch, daß die Mitglieder der Gruppe nichts weiter in der Hand haben als ihr ethniespezifisches Kapital, das seinen Wert komplett verlieren würde, wenn die jeweilige Gegenseite obsiegen würde. Es geht, kurz gesagt, wirklich um alles oder nichts - und nicht bloß um die Durchsetzung ansonsten belangloser virtueller Konstruktionen.

Solche Verhältnisse entstehen vorzugsweise in $\mathrm{Pe}$ rioden der gesellschaftlichen Transformation und

ökonomischen und politischen Hintergründe der Konflikte in - dem ehemaligen - Jugoslawien vgl. den Beitrag von Galic (1996: $60 \mathrm{ff}$. insbesondere). 
des Zusammenbruchs eingelebter Ordnungen und Interdependenzen, einschließlich solcher der „Modernisierung" und der damit verbundenen Bedrohung eines gruppenspezifischen Kapitals gegen seine universalistische Entwertung. Wenn dabei nicht nur eine $A b-$, sondern sogar eine drastische $U m$ wertung des spezifischen Kapitals droht, derart, daß das, was vorher, etwa die schwarze oder die weiße Hautfarbe, eine Stasi-Medaille oder das Bundesverdienstkreuz, die Zugehörigkeit zur serbischen, zur kroatischen oder zur muslimischen Volksgruppe etwa, Privilegien, Einkommen, Prestige und Macht brachte, nunmehr in Arbeitslosigkeit, Schande, Verfolgung oder gar Tod zu mutieren droht, dann wird diese strukturelle Grundlage des Konfliktes ganz besonders virulent. Sie ist das strukturelle Motiv für alle daran anschließenden folgenden symbolischen "Identitätskämpfe“.

Hat dieser Konflikt einmal offen begonnen (siehe dazu gleich noch unten) und gibt es keine mäßigenden Mechanismen, wie Abstimmungsregeln, übergreifende universalistische Kriterien, gemeinsame Interessen oder gar ein funktionierendes Herrschaftszentrum, verlagert sich der Kampf um die Verfassung bald auf die möglichst vollständige Okkupierung der „letzten“ Mittel, die es gestatten, die jeweilige Verfassung zu ändern oder zu sichern. Das sind im Falle der ethnischen Konflikte die politische Herrschaft und damit letztlich genügende militärische Mittel. Obsiegen kann bei diesem Kampf um die politische Herrschaft aber immer nur eine Partei: Herrschaft ist ein typisches Positionsgut, das nur dem nutzt, der es als erster kontrolliert, insbesondere weil die Herrschaft es erlaubt, die verfassungsdefinierenden Mittel immer weiter zu kumulieren. Und genau deshalb ist der Wettlauf um die verfassungsdefinierende Herrschaft so gnadenlos und unvermeidlich - wenn er denn einmal begonnen hat und Rücksichten nicht weiter genommen werden müssen.

\section{Ethnisches Framing}

Mobilisiert, organisiert und bestritten wird dieser, wie man leicht sieht: letztlich außerordentlich „materiell“ unterbaute, Konflikt mit allerlei Überbau-Codierungen einer kollektiven Identität der jeweiligen Gruppen, die oberflächlich gesehen wie kulturelle Maskeraden aussehen, es jedoch keineswegs sind: Es ist ein Kampf um zentrale, nicht nur als Schimıären oder luftleere Konstruktionen vorhandene, „Werte" und um deren „Anerkennung", und eben nicht bloß um die Anerkennung irgend- eines ansonsten kostenlosen symbolischen Konstruktes einer an sich folgenlosen und bloß in Leere „konstruierten“ kollektiven Identität. Das geschieht zunächst mit nicht minder kämpferisch gemeinten Versuchen, für die jeweilige Gruppe in einer angenommenen Öffentlichkeit Resonanz zu finden oder mit anderen Gruppen Koalitionen zu bilden, insbesondere dadurch, da $\beta$ gezeigt werden soll, da $\beta$ das jeweilige Anliegen schon a priori besonders vortrefflich und von Interesse auch für andere sei, daß die jeweilige eigene Gruppe die grundlegend bessere Lebensweise repräsentiere und die jeweils bekämpfte Gruppe grundsätzlich minderwertig wäre. Es ist der Vorgang, der in der Bewegungsforschung inzwischen als Framing bezeichnet wird und für den es in den, vor allem: neuen, sozialen Bewegungen häufig sogar, mehr oder weniger strategisch agierende, „Unternehmer" gibt. Das sind vorzugsweise die jeweiligen Eliten, die über Medieneinfluß versuchen, das Framing für die jeweilige eigene Sache begünstigend zu steuern, namentlich durch Verweis auf ein allgemeineres Interesse an der partikularen Sache oder auf eine lange Vorgeschichte, die jetzt endlich auf Erfüllung dränge. Dieses Framing ist dabei kein bloßes Beiwerk, sondern ein gewichtiger Teil, oft sogar die Bedingung, der Mobilisierung und der „Bewegung“ der Bewegung (siehe dazu noch gleich unten mehr). Es dient der Gewinnung von Anhängern in einer noch unbeteiligten Öffentlichkeit und der Mobilisierung der jeweils eigenen Gruppe, die ja auch bei ethnischen Konflikten nicht sofort immer mit Begeisterung dabei ist und vor dem Problem des abwartenden free riding steht, auf das vor langer Zeit Mancur Olson als nicht leicht zu lösendem Mobilisierungshemmnis hingewiesen hat. Erleichtert wird der Vorgang des öffentlichen Framings dadurch, da $B$ es gelegentlich latente, mit historischen Erfahrungen verbundene, teilweise mythologisch zugespitzte, sozial geteilte fertige „Modelle“ der Abgrenzung, der Distanz, der Abwertung und der Auseinandersetzung nach außen und des Wir-Gefühls, der kollektiven Identifikation, der Solidarität und der Vortrefflichkeit nach innen gibt, die bei dem Akt des strategischen Framings in der Öffentlichkeit dann nur noch ,aktiviert" werden müssen oder wenigstens den ersten Anhaltspunkt für ihre weitere Zuspitzung abgeben.

Der Erfolg eines die Situation definierenden ethnischen Framings ist mit den Versuchen dazu natürlich noch nicht garantiert. Denn warum sollten die „Massen“ bzw. die Öffentlichkeit den Eliten und den Medien, die deren Botschaften transpor- 
tieren, in den angebotenen Codierungen eigentlich folgen? Spätestens hier zeigt sich, daß die Durchsetzung von Codierungen und Frames auch etwas mit Selektionen zu tun hat, die die Akteure vornehmen, aus denen sich die „Öffentlichkeit" zusammensetzt, und zwar durchaus als „Entscheidung“ zwischen verschiedenen Alternativen, die von den Framing-Unternehmern, den Eliten oder sonstwie angeboten werden. Denn wie sollte es sonst zu irgendeiner sozialen Verbreitung der Situationsdefinitionen in einer Öffentlichkeit kommen? Die „Wirkung“ einer jeden kommunikativen Mitteilung besteht ja in mindestens der Rezeption derselben. Und das ist immer eine „Selektion“: Annahme oder Ablehnung, wie uns Niklas Luhmann lehrt.

Der um das Konzept des Framings vervollständigte RC-Ansatz gibt zu dieser rezeptiven Selektion zwei, aus der Wert-Erwartungstheorie abgeleitete Bedingungen an (vgl. dazu Esser 1996c): Es muß erstens solche gedanklichen rahmenden Modelle, wenigstens in rudimentärer Form, bereits vorher als sozial geteilte Bezugsrahmen geben: Ethnizität läßt sich aus dem Nichts heraus nicht so einfach nur „konstruieren“. Eine irgendwie erinnerbare "Geschichte“ von Konflikt und Abgrenzung nach außen bzw. der Gemeinschaftlichkeit und der Solidarität nach innen muß es schon geben, an die das Framing anknüpfen könnte. Und es muß dann auch noch gelingen, die Frames zu aktivieren und zu verstärken, etwa durch, auch symbolisch dramatisierte, Hinweise auf typische Untaten der jeweiligen Gegenseite und typische, auch im ,allgemeinen" Interesse liegende Vortrefflichkeiten des eigenen Anliegens. Das ist die ,argumentative“, die „kulturelle“, die "symbolische“, die kognitive Seite des Framings und der kommunikativen $\mathrm{Re}$ zeption. Es muß zweitens mit der Übernahme des jeweiligen Frames aber auch ein bestimmter Anreiz verbunden sein. Mindestens dürfen mit der Übernahme des Frames und dem damit verbundenen Handeln keine allzu großen Kosten verbunden sein. Das ist die bewertende Seite des Framings und der kommunikativen Rezeption von angebotenen Codierungen. Die durch die Frames meist auch ausgelösten Emotionen kombinieren dabei die kognitiven und die bewertenden Elemente.

Aus der Sozialpsychologie der Bildung kollektiver Identitäten weiß man zwar, daß eine gewisse rahmende Grenzziehung durchaus auch schon ohne besonderes Dazutun und ohne Bezug auf solche vorgefertigten, historisch verankerten Framing-Modelle erfolgt, nämlich alleine schon als Folge der bloßen Kategorisierung, etwa durch eine Einteilung von individuellen Akteuren in arbiträr gebilde- te Gruppen (vgl. Tajfel/Turner 1986). Aber die rahmende Kraft dieser "minimalen" Kategorisierungen ist nur schwach, wenn sie nicht durch "materielle“ Interessen unterstützt ist oder durch symbolische Akte entfacht wird, und sie verfällt sofort, wenn gewisse Interessen hinzutreten und die kategorialen Gruppengrenzen überkreuzen.

Ein erfolgreiches ethnisches Framing bei einer abwartenden Öffentlichkeit ist nach der WE-Theorie nur dann zu erwarten, wenn beide Bedingungen erfüllt sind: Die kognitive Sicherheit der Geltung und die bewertenden Anreize dafür oder wenigstens nicht massiv dagegen.

Alle Versuche, gewisse Symbole oder Codierungen für nicht schon sicher geltende Rahmungen durchzusetzen, scheitern danach, wenn deutlichere Anreize dem entgegenstehen. Nicht ganz fest verankerte Frames wirken, wie nicht strikt "unbedingte" moralische und sonstige "Einstellungen" ganz allgemein, daher vor allem (oder gar: nur) in low-cost-Situationen (vgl. dazu auch die neuerlichen Hinweise bei Diekmann/Preisendörfer 1998). Bei hohen Kosten sind die Symbole und "kommunikativen“ Versuche zur Durchsetzung von Frames nichts als cheap talk, wie die Ökonomen sagen. Aber wenn ein rahmendes Modell der Abgrenzung bei den Akteuren einmal mit Sicherheit "gilt", etwa weil es deutlich sichtbare, „signifikante" Akte des Konfliktes und der Feindseligkeit gegeben hat, provoziert vielleicht durch isolierte Aktionen kleiner Einheiten, dann zählen die Kosten des Konfliktes in der Tat nichts mehr (vgl. dazu auch die Modellierung bei Weingast 1998: 168f.). ${ }^{3}$ Sie werden in der nun alles als „Konflikt" rahmenden Orientierung in den Hintergrund gedrängt. Es ist genau das, was den Anschein der Irrationalität und der Expressivität der Abläufe nahelegt und dafür sorgt, daß es schließlich tatsächlich nur noch ,irrational" und expressiv zugeht.

Bei ethnischen Konflikten sind diese beiden Bedingungen häufig erfüllt, ganz anders als bei bloBen kulturellen Stilfragen ohne jeden materiellen Hintergrund und auch ganz anders als bei den bloß materiell motivierten Klassenkonflikten ohne besondere kulturelle Rahmung: Es gibt die sozial geteilten gedanklichen rahmenden Modelle, etwa aus (wie verblaßt auch immer erinnerbaren) ethnischen Mythen, wenigstens latent. Und es gibt wegen der Spezifizität des Kapitals, um dessen Bewertung es bei dem Konflikt jeweils geht, auch deutliche kollektive Anreize zur Übernahme des Frames, die um so stärker sind, je weniger ,generalisierbares“, an die Existenz der ethnischen

${ }^{3}$ Vgl. dazu den interessanten, mit dem hier entwickelten Modell in den Grundzügen vollauf vereinbaren Beitrag von Bergmann (1998) über Pogrome. Daß hierbei Bezugsgruppenprozesse und die symbolisch-interaktive „Produktion" von Anerkennung in subkulturellen Milieus eine wichtige Rolle spielen, sei nur am Rande noch erwähnt; vgl. dazu näher: Esser (1999, Kapitel 50 und 51). 
Gruppe eben nicht gebundenes, Kapital auch noch kontrolliert wird, wie etwa ökonomische Ressourcen, Humankapital oder gewinnbringende ,weak ties" über die Grenzen der ethnischen Gemeinschaft hinaus.

Die Eskalation des Konfliktes wird bei einem einmal angelaufenen ethnischen Framing nahezu unvermeidlich, wenn sich die Gruppen gegenseitig nicht dominieren können und wenn es keine externen Garantien gegen die Ausnutzung einer einseitigen Zurückhaltung gibt. Weil jetzt die Situation mehr und mehr unter der Perspektive des Konfliktes gesehen wird, werden feindselige Akte der Gegenseite nicht mehr für unmöglich gehalten, selbst wenn es vorher ein freundliches Miteinander gab. Und daher wird mit einer auch nur geringen Änderung der Erwartungen und der Rahmung bald der möglichst rasche präventive Gegenschlag ,rational", der - tragischerweise - keineswegs aggressiv gemeint sein muß, sondern nur die nun als zunehmend wahrscheinlicher angesehene Opferposition verhindern soll. Es ist der - von Interessen strukturell untermauerte, von symbolisch bewerteten Aktionen eingeleitete und dann auch von Befürchtungen getragene - Umschlag der Definition der Situation von irgendeinem vertrauensvollen modus vivendi der Gruppen in den Hobbesschen Krieg aller gegen alle, aus dem es, bekanntlich, nur unter sehr speziellen institutionellen Umständen wieder einen Ausweg gibt (vgl. dazu auch Weingast 1998: 172 ff.).

Wenn es nun deutliche Grenzen zwischen den Akteuren eines Kollektivs in der Ausstattung mit nur spezifischem Kapital gibt, und wenn eine Situation der institutionellen Anomie und der gesellschaftlichen Transformation besteht, dann sind in verstärktem Ausmaß Versuche zu erwarten, die jeweilige Verfassung so zu gestalten, daß das jeweilige spezifische Kapital seinen Wert behält oder gar erst gewinnt. Sofern nun, zunächst auch nur schwach verankerte, „kulturell“ definierte Identitäten mit den Grenzziehungen in der Ausstattung mit spezifischem Kapital und den dadurch erzeugten strukturellen Konfliktlinien übereinstimmen, und sofern es gar auch organisatorische und soziale Beziehungen entlang dieser Grenzen gibt, gewinnen die zunächst nur latenten kulturellen Modelle von kollektiven Identitäten eine hohe Plausibilität und auch dadurch eine hohe emotionale Bindekraft. Darauf hatte schon Max Weber hingewiesen, als er von dem ,gemeinsamen Schicksal" als dem strukturellen Hintergrund aller kultureller ethnischer Gemeinschaftskonstruktionen sprach.

\section{Das Problem der Mobilisierung}

Mit den bloßen Interessen an den Versuchen zu einer Verfassungsänderung oder -sicherung ist eine „Bewegung“ bekanntlich noch lange nicht mobilisiert. Es gibt immer ein free-rider-Problem, das um so gravierender ist, je größer die Gruppe der „Betroffenen" ist. Es gibt bekanntlich verschiedene Auswege aus diesem Problem: Die „Organisation" der Bewegung, Netzwerke und ,soziales Kapital" sowie ein moralisches Framing der kollektiven Identität und der guten gemeinsamen Sache.

Von großer Wichtigkeit scheinen bei der Mobilisierung ethnischer Konflikte, besonders wenn die weitere Öffentlichkeit noch abwartend ist, kleine, stabile, gut geführte, in lokalen Netzwerken verankerte und als „lebensweltliche“ Bezugsgruppen fungierende, „radikale" Kerntruppen zu sein, die sowohl die Organisation der Bewegung besorgen wie mit auch strategisch und provokativ geplanten Aktionen den Framingproze $B$ zu starten und zu tragen vermögen und immer wieder neu anheizen können. Zu Hilfe kommt solchen Aktionen die inzwischen weltweite Aufmerksamkeit über die Medien.

Aber auch die stärkste Resonanz in der medialen Öffentlichkeit reicht oft für die Mobilisierung nicht aus, zumal dadurch auch Gegenmobilisierungen gerahmt werden können. Nun muß es noch andere Gruppen mit gewissen, durchaus auch anders motivierten, Bereitschaften an der Bewegung geben, die auf diese agents provocateurs, sozusagen, nur gewartet haben, weil sonst der Proze $B$ zwar anfängt, aber dann auch bald wieder stecken bleibt. Ohne solche, in einer breiteren Öffentlichkeit schon vorbereiteten Bereitschaften - und Fähigkeiten! - zur Teilnahme versanden soziale Bewegungen bald, von denen die ethnischen Konflikte ja nur ein Spezialfall sind.

Die Überwindung des free-rider-Problems ist für ethnische Gruppen mit wenigstens rudimentären Formen von Organisationen und Netzwerken entlang der Gruppengrenzen und mit mindestens Resten ethnisch definierter kollektiver Identifikationen und Solidaritäten, wie man sieht, schon strukturell leichter als für andere Aggregate, die sich ausschließlich auf der Basis gemeinsamer Interessen zusammenfinden, wie das bei den sog. Klassenkonflikten der Fall ist oder war (vgl. dazu etwa Hechter et al. 1982). Diese kulturellen und askriptiven Bedingungen der Mobilisierung hat Karl Marx wohl mit seinen vorwiegend "materialistischen" Hypothesen über den Zusammenhang der Stärke von Klasseninteressen und der Mobilisierung von Revolutionen ubersehen. Mancur Olson hat ihn in der Unterschätzung des Mobilisierungsproblems auch zu Recht kritisiert. Heute wissen wir, daß Netzwerke, zunächst meist zu anderen Zwecken eingerichtete Organisationen und ethnisches Framing beste Bedingungen für die Überwindung des Problems des kollektiven Handelns 
sind. Und daher verwundert auch kaum, daß es gerade die ethnischen Konflikte sind, die sich im Zuge der vielen Transformationen und Ungleichzeitigkeiten beim Prozeß der Modernisierung der Welt bemerkbar machen (vgl. dazu näher Esser 1988: $241 \mathrm{ff}$.). Aber, und das sei vorsichtshalber auch wieder in Erinnerung gerufen, ohne den "materiellen" Konflikt über die Bewertung des spezifischen Kapitals bleiben die ethnischen Milieus, Organisationen und Identitäten und die Versuche der Durchsetzung ethnischer Codierungen folgenlos. Der Konflikt um die Bewertung spezifischer Kapitalien ist aber ein solcher materieller Konflikt, in Phasen des gesellschaftlichen Umbruchs, wie es die Vorgänge der Modernisierung immer gewesen sind, der wohl gravierendste sogar. Er könnte erst mit der weiteren Modernisierung, funktionalen Differenzierung und Universalisierung der Welt und mit der damit verbundenen universellen Ausweitung der Kontrolle von generalisierbarem Kapital zu einem Ende kommen. Aber davon ist die Welt (noch?) weit entfernt, und wird es wohl, trotz aller Globalisierungsrhetorik, auch (vorerst?) bleiben.

Diese Vorgänge finden selbstverständlich allesamt als Proze $\beta$ statt, ausgehend unter Umständen von relativ unbedeutenden Anlässen, bei dem sich Akteure oder Kollektive in Interaktion miteinander befinden und sich im Vollzug ihres Tuns gegenseitig beobachten und, oft unbeabsichtigt, aber durchaus auch strategisch, anzeigen, welches gedankliche Modell - etwa: Konflikt oder Kooperation, Ausgrenzung oder Integration - für ihre Beziehungen wohl gerade gilt oder gelten soll. Und sie finden, natürlich, auch gelegentlich vor den Augen einer das Geschehen beobachtenden Öffentlichkeit statt, die als "dritte“ Partei wahrgenommen und zu beeinflussen gesucht wird. Die - mehr oder weniger: strategischen - Interaktionen der konfligierenden Parteien untereinander und mit der Öffentlichkeit als „Beobachter" des Streits sind dann als Sequenzen von Situationen und Situationsubergängen, auch als solche von Prozessen des „Aushandelns", der Bildung von Koalitionen oder auch einer stetigen Oszillation von Schwerpunktverschiebungen in der Dominanz der einen oder anderen Codierung modellierbar.

Diese Modellierung beginnt naturlich immer mit einer angenommenen Ausgangssituation und den damit verbundenen materiellen Strukturen, etwa der jeweiligen Ausstattung mit spezifischem und generalisiertem Kapital bei den Gruppen, den bestehenden institutionellen Regelungen, etwa der aktuellen Geltung einer bestimmten Verfassung oder einer Situation der institutionellen Transformation, und den jeweils - latent - verankerten gedanklichen Modellen kollektiver Identitäten, von denen die rahmende kulturelle Definition der Situation ausgeht. Das Tun der Akteure hat dabei nicht nur materielle Folgen, sondern, weil sich die Akteure beobachten oder irgendwie sonst mitbekommen, was geschieht, unver- meidlicherweise auch symbolische Konsequenzen: Handeln ist immer auch ein - mehr oder weniger ,signifikantes" - Zeichen für die „Geltung" des einen oder des anderen Modells der Definition der Situation. Die Symbolik der Handlungen verändert dabei, technisch gesehen, die sog. second-order-probabilities bei unsicheren Erwartungen derart, daß diese Erwartungen über die Geltung bestimmter Rahmen nun sicherer werden als zuvor. Darauf verändert sich, etwa durch einen offensiven und unmißverständlichen Akt der Feindseligkeit, wiederum die Rahmung der Situation bei den Akteuren, die daraufhin wieder etwas tun, und so weiter, so daß auch Kaskaden der Mobilisierung und der Zuspitzung und Radikalisierung des interaktiv ablaufenden Framings entstehen können, bei denen die Akteure selbst kaum noch verstehen, was mit ihnen geschieht und was früher mit ihnen war, Prozesse, die so auch formal modelliert werden können, etwa über Diffusionsmodelle oder über solche des Rustungswettlaufs.

Man könnte diesen Vorgang der symbolisch-interaktiv erzeugten und dann kollektiv geltenden Definition der Situation auch als soziales Framing oder als soziale Konstitution eines Bezugsrahmens bezeichnen (siehe dazu auch noch Abschnitt 3 unten).

Mobilisierungen tuber Prozesse des sozialen Framings oder andere Formen der interaktiven „Ansteckung“ sind meist alles andere als „,deterministisch" und „linear". Denn auch wenn alle ,günstigen" Bedingungen eines salienten Framings bei einem Aggregat von interessierten Akteuren erfüllt sind, muß es immer noch keineswegs zu einer erfolgreichen kollektiven Verbreitung des Framings und zur Mobilisierung der weiteren Öffentlichkeit kommen: Es gibt stets auch noch das Problem der „kritischen Massen“ - strukturierte Unterschiede in den latenten Bereitschaften und Fähigkeiten zur Teilnahme bei den verschiedenen Gruppen einer Öffentlichkeit, modellierbar als unterschiedlich hohe Schwellenwerte, sich der Bewegung anzuschließen bzw. die Codierungsangebote anzunehmen oder nicht. Und es gibt immer auch Unterschiede in der Struktur der sog. Produktionsfunktionen der Bewegung, die schon technisch festlegen, bei welchem Einsatz von Mitteln eine Bewegung Erfolg hat oder nicht.

Kurz: Jetzt werden die Struktur der technischen Produktionsfunktionen und die Struktur der sozialen Verteilung der latenten Bereitschaften bzw. der Schwellenwerte zur Übernahme der entsprechenden Codierungen in Abhängigkeit der bereits mobilisierten anderen Akteure und die damit gegebenen, strukturell festgelegten Anschlußmöglichkeiten für einen Diffusionsproze $B$ wichtig, so wie das Mark Granovetter (1978), Thomas C. Schelling (1978) oder Pamela Oliver et al. (1985) schon vor langer Zeit gezeigt haben (vgl. auch Lohmann 1994). 
Das alles sind Gegebenheiten, die nicht unter der Kontrolle einzelner Akteure stehen. Mit den damit oft verbundenen „Nichtlinearitäten“ beim Prozeß der Mobilisierung läßt sich gut (weil: $k a u$ sal) erklären, warum es manchmal oft nur winzige Lücken und unscheinbare Umstände sein können, die einen Mobilisierungsproze $\beta$ in die eine oder die andere Richtung drängen können, warum es manchmal ganz unweigerlich zur kompletten Mobilisierung kommt, warum sich manchmal, obwohl alles vor Bereitschaft nur so bebt, aber auch gar nichts tut, und warum das alles auf eine ,lineare", „, deterministische" und variablensoziologische Weise, etwa mit dem bloß ,statistisch“" aggregierten Interesse an einer Bewegung, in der Tat nicht zu erklären ist.

Die Rahmungsversuche der Rahmungsunternehmer in der beobachtenden Öffentlichkeit sind dann als ein Kampf um die Erhöhung oder Absenkung der Schwellenwerte bei „strategisch“ für den Erfolg der Bewegung als wichtig angesehenen Gruppen interpretierbar - mit kollektiven Folgen, die von den dadurch, auch unbeabsichtigt, bewirkten Änderungen in den Schwellenwerten und den dann gegebenen Verteilungen der Schwellenwerte, sowie von den Produktionsfunktionen für die Mobilisierungsressourcen abhängen. Und weil niemand als Einzelner die Struktur der Produktionsfunktionen und die Verteilungen der Schwellenwerte und der Effekte des Framings komplett kennen oder gar beherrschen kann, gibt es in der Tat immer wieder freudige oder weniger freudige Überraschungen über den Lauf der Dinge, nicht nur bei den Rahmungsunternehmern.

\section{Die Eigendynamik ethnischer Konflikte}

Auf diese Weise wird die oft zu beobachtende, die Akteure auch immer wieder überraschende und in mancherlei Fallen verstrickende Eigendynamik sozialer Bewegungen und ethnischer Konflikte gut verständlich und methodologisch angemessen erklärbar. Der RC-Ansatz gibt mit seiner "Logik“ schließlich auch eine einfache Antwort darauf, warum es, wenn der Vorgang einmal angelaufen ist, aus den eigendynamischen Kaskaden des Konfliktes - zunächst wenigstens - keinen ,endogenen" Ausweg gibt, selbst wenn alle das wollten: Wer zuerst mit dem einmal begonnenen Konflikt aufhört, hat schon alles verloren. Und deshalb wird in einer unerbittlichen Pfadabhängigkeit weitergemacht, zumal sich mit der Fortführung des Konfliktes die kooperativen Alternativen immer weiter ausschließen - meist bis zu einem nicht aufhören wollenden bitteren Ende. Die Beendigung des Konfliktes ist nämlich, schon wieder einmal, ein prisoners dilemma mit der wechselseitigen Defektion als dominante Strategie und einem paretoinferioren Gleichgewicht, wie es im spieltheoretischen Jargon so schön heißt, und ein neues Problem des kollektiven Handelns - diesmal das der Einigung der Konfliktparteien auf eine Beendigung des Konfliktes.

Aber auch für ein ,endogenes" Ende solcher Konflikte gibt der RC-Ansatz systematische, logische, rationale und deshalb verständliche Hinweise. Weil der Konflikt, besonders wenn die Unterstützung von außen ausbleibt, schließlich alle Parteien merklich ruiniert und auch die Ressourcen, um deren Bewertung der Kampf tobt, aufzuzehren droht, wird schlieBlich nicht mehr das vorzeitige einseitige Aufgeben, sondern der Sturz in den gemeinsamen Untergang zur schlimmsten Alternative - für beide Parteien. Technisch gesehen wandelt sich die Situation von einem Nullsummenspiel in ein Negativsummenspiel und darüber von einem prisoners dilemma in ein chicken game (vgl. dazu Taylor 1987: Kapitel 2; Esser 1999: Kapitel 20: Strategische Situationen). Und ein chicken game hat bekanntlich zwei, und zwar: instabile, Gleichgewichte. Aber gerade wegen dieser Instabilität kann es jetzt einen Ausweg aus dem unerbittlichen Pfad in die gemeinsame Selbstzerstörung geben: Statt des immer weiter gesteigerten Konfliktes gibt es nun einen nicht enden wollenden Wechsel zwischen Verhandlungen, Vereinbarungen, Waffenstillständen - und deren ständiger Verletzung. Ein Ende wird, wenn nicht noch anderes geschieht, zwar erst durch externe Eingriffe oder übergreifende Rücksichten möglich. Aber auch dann bleibt die Situation dauerhaft in der Schwebe, wie wir das seit einiger Zeit quer durch die Welt fast täglich im Fernsehen beobachten können.

Gibt es aber gar, woher auch immer, deutliche, ,positive" gemeinsame Interessen, etwa: die Verbündung gegen einen plötzlich auftauchenden gemeinsamen Feind, die Entdeckung von nur gemeinsam ausbeutbaren Bodenschätzen, unter der Hand entstandene wirtschaftliche und politische Interdependenzen, dann wandelt sich das "Spiel“" in das schon sehr viel freundlichere assurance game, das jetzt "nur" noch ein mildes Vertrauen in die „Vernunft" des anderen voraussetzt, damit es zur Kooperation und zur Beendigung des Konfliktes kommt. Leider erzeugen Konflikte endogen solche „positiven" gemeinsamen Interessen nicht, sondern nur das „negative" Interesse an der Vermeidung des gemeinsa- 
men Untergangs. Und genau deshalb kann man sich auch nicht darauf verlassen, daß ein einmal angelaufener und etablierter Konflikt - sozusagen - einmal von alleine aufhört.

Kombinieren sich dagegen die beiden Interessen das negative der Furcht vor dem gemeinsamen Untergang und das positive an gewissen gemeinsamen Projekten -, dann entsteht sogar eine deutliche Interessenkonvergenz. Hier bildet sich, wie beim prisoners dilemma, ein stabiles Gleichgewicht in dominanten Strategien, diesmal aber eines der beiderseitigen Kooperation. Und das ist jetzt, gänzlich anders als beim prisoners dilemma, paretooptimal. Spätestens dann kommt es auch zu einem Wechsel in den kulturellen Rahmungen, die dann bald auch mit allerlei Symbolen und Ritualisierungen und dem Mythos ausstaffiert werden, daß die ehemaligen Erbfeinde eigentlich ja immer schon geborene Freunde gewesen wären, und daß die ganze feindselige Vorgeschichte nur ein furchtbarer Irrtum gewesen sei.

\section{Das Modell der soziologischen Erklärung und das Konzept der Situationslogik}

Die geschilderten Vorgänge skizzieren die Art der Erklärung ethnischer Konflikte im Rahmen des um gewisse kognitive bzw. kulturelle Komponenten vervollständigten $R C$-Ansatzes, gegen den sich Eder und Schmidtke wenden. Es ist eine Anwendung des ansonsten in seinen Bestandteilen vollkommen unveränderten Modells der soziologischen Erklärung, das im elementaren Fall, wie in zwischen allgemein bekannt sein dürfte, aus der Modellierung der jeweiligen Logik der Situation, der Anwendung einer (nomologischen) Handlungstheorie zur Erklärung des situationsorientierten Handelns (bzw. des Framings) der Akteure und aus einer aggregierenden Ableitung gewisser struktureller Effekte besteht. Den einfachen Dreier-Schritt einer elementaren soziologischen Erklärung kann man natürlich in beliebige Konstellationen der simultanen „Interaktion“" von Akteuren und in beliebig lange und differenzierte Sequenzen nach vorne und nach hinten verlängern, und es können auch beliebige Anzahlen - zwei, drei, viele - und beliebige Aggregationen von Akteuren - Individuen, Kategorien, Aggregate, kollektive und korporative Akteure - berücksichtigt werden (vgl. dazu ausführlich Esser 1993: Teil B: Soziologische Analysen und Erklärungen).

Die bei den soziologischen Erklärungen dabei inzwischen bevorzugte Wert-Erwartungstheorie ent- hält zwei verschiedene Konstrukte: Erwartungen und Bewertungen. Sie sind als "Interaktionseffekt" der Erwartungen mit den Bewertungen verbunden. Das ist keine, wie manche offenbar meinen, überflüssige Präzisierung. Es ist erstens der - unverzichtbare - allgemeine (nomo-)logische Kern des Erklärungsarguments. Und es ergeben sich zweitens daraus alleine schon eine Reihe von nicht-trivialen Aussagen, die manches verständlich machen, was sonst nur als „Paradoxie“ gesehen werden kann: Die Auflösung des sog. TocquevilleParadox etwa, wonach Revolutionen gerade nicht bei völliger Verelendung ausbrechen, sondern erst dann, wenn sich die Restriktionen auch für weitere Teile der Bevölkerung schon zu lockern beginnen. Oder die Antwort auf die Frage, warum es keinen unmittelbaren Zusammenhang zwischen - auch massiven - ethnischen Schichtungen und ethnischen Konflikten gibt.

Die „kognitivistische“ Vervollständigung der RC-Theorie besteht dann in einer einfachen Erweiterung dessen, was unter „Handlungen“" zu verstehen ist: Es gehört dazu auch das, wie Max Weber sagt, ,innere Tun“, also: die Wahrnehmung und die „Einstellung“ auf die Situation, wovon das Framing, die subjektive Definition der Situation mit dem daran anschließenden Handeln, nur ein Sonderfall ist. Auf diese Weise lassen sich - unter anderem - das wertrationale, das affektuelle und das traditionale Handeln der Typologie von Max Weber, auch die anderen, neben den von Max Weber unterschiedenen weiteren „Typen“ des Handelns, wie das ,normative", das „dramaturgische“ und das "kommunikative“ oder auch das "kreative“ Handeln als Spezialfälle des allgemeinen Modells der Wert-Erwartungstheorie rekonstruieren. Und auch die „kognitivistische" Bildung von „beliefs“, wie sie Raymond Boudon (1996) vorschlägt, läßt sich so erklären (vgl. Esser 1999: Kapitel 47: Die Selektion des Bezugsrahmens): Es werden jeweils die „beliefs" selegiert, die sich angesichts der bisherigen Glaubens-Routinen und der gegebenen Möglichkeiten der "rationalen“ Durchdringung des Geschehens als die vergleichsweise angemessensten erwiesen haben. Die „RC+“-Erweiterung ist im übrigen die theoretische Antwort auf die meisten der gegen die einfache WE-Theorie als entscheidungstheoretischem Kern vorgebrachten Einwände, etwa auch den, daß das normative, das affektuelle oder das wertgeleitete Handeln damit nicht erklärbar wären. Wenigstens was die dabei immer wieder vorgebrachten Experimente von Tversky und Kahneman zum „Framing“ angeht, ist zudem keineswegs ausgemacht, ob es diese „Anomạlien“ wirklich gibt (vgl. Stoké 1998 und einige andere Beiträge in Druwe und Kunz 1998).

Mit der Nutzung dieser handlungstheoretischen Präzisierung geht es bei den soziologischen Erklärungen aber keineswegs primär um das Verhalten von "Individuen“, sondern um die Erklärung der Genese emergenter struktureller Effekte, ein- 
schließlich der oft komplizierten und durchaus "nicht-linearen" und „nicht-deterministischen" Eigendynamik von Mobilisierungskaskaden und deren Beendigung, wie sie oben beschrieben wurde.

Die WE-Theorie fungiert im Modell der soziologischen Erklärung also nur an einer, in der Tat gänzlich ,unsoziologischen“, Stelle der kausalen Umsetzung der situativen Strukturvariablen in das Handeln der Akteure, von dem alle strukturellen Folgen abhängen. Sie dient nur als Logik der Selektion, nur als „decision theory“ für die momentane Handlung, aber eben nicht schon alleine als irgendeine Erklärung der weiteren Folgen des Geschehens und der erneuten Strukturierung der Situation. Sie ist jedoch unerläßlich, "damit" die Eigendynamiken in der Überführung der Strukturen in das Handeln und dessen strukturelle Folgen keine „logische“ Lücke haben.

Kurz: Die WE-Theorie ist notwendig, aber in keiner Weise hinreichend für eine angemessene soziologische Erklärung ohne logische Lücke. Ihre Verwendung erzwingt dabei eben nicht, die Erwartungen und Bewertungen der Akteure als „subjektiv“" im Sinne irgendeiner Beliebigkeit anzusehen. Vielmehr können ihre zentralen Konstrukte, die Erwartungen und die Bewertungen, problemlos mit den „objektiven“ Umständen verbunden werden.

Die theoretische Verbindung zwischen den objektiven Parametern der Situation und den - letztlich natürlich immer: subjektiven - Erwartungen und Bewertungen der Akteure geschieht bekanntlich über die sog. Brückenhypothesen, etwa derart, daß eine Gruppe mit nur spezifischem Kapital objektiv einen besonders hohen Anreiz hat, die Verfassung einer Gesellschaft zu ihren Gunsten zu ändern. Diese Brückenhypothesen müssen nur die jeweils "typischen" und „relevanten“ Situationsmerkmale beschreiben und nicht jeden Grashalm individueller Ideosynkrasien. Was „typisch“ und was „relevant" ist, können die Akteure meist selbst nicht wissen. Daher sind narrative Interviews, hermeneutische Verfahren oder standardisierte Motivanalysen zu ihrer Gewinnung allenfalls heuristisch geeignet (siehe dazu auch noch gleich unten zum Problem des Psychologismus). Das Geschäft der Formulierung der Brückenhypothesen muß schon der das Geschehen aus der Vogelperspektive objektiv „beobachtende" Sozialwissenschaftler selbst besorgen. Das kann er aber meist auch schon bald, wenn er nur die grundlegenden sozialen Produktionsfunktionen, die primären Zwischengüter, die kulturellen Ziele, die funktionalen Imperative, die Codes und die Verteilung der indirekten Zwischengüter bzw. der institutionalisierten Mittel in einer Gesellschaft kennt (vgl. zum Konzept der sozialen Produktionsfunktionen und der ,objektiven" Strukturierung der subjektiven „Definition der Situation“: Esser 1996c: 5 ff.).

Die Motive und das Wissen der Akteure sind nach dem Konzept der soziologischen Erklärung also immer sozial und immer objektiv strukturiert. Und die sozialen Strukturen und Prozesse sind immer auch nur als über die Absichten der Akteure hinausweisende und manchmal nur sehr komplex aggregierbare, strukturelle Folgen des Handelns der Akteure versteh- und erklärbar.

Das Programm der so gefaßten soziologischen Erklärung ist nun aber nichts anderes als die Ausarbeitung, Verfeinerung und Aktualisierung jenes Konzeptes, das Karl R. Popper einst als Situationslogik bezeichnet und deutlich vom sog. methodologischen Psychologismus abgegrenzt hatte. ${ }^{4}$

Auf den ersten Blick klingt die Position Poppers etwas verwirrend und „widersprüchlich“ und wird deshalb auch oft mißverstanden. Danach lassen sich die verschiedenen sozialen Prozesse nicht schon aus gewissen "subjektiven“ Motiven der Menschen heraus „psychologisch“, sondern nur „soziologisch" erklären. Zwar seien für die Erklärung situationslogischer Prozesse der Bezug auf gewisse psychologische Annahmen, wie etwa über das rationale Handeln, nötig. Insofern komme - statt der Suche nach holistischen Strukturgesetzen - für eine erklärende Soziologie auch nur ein „Methodologischer Individualismus“ in Frage, der das soziale Geschehen eben nicht ,aufgrund sogenannter 'Kollektive' (Staaten, Nationen, Rassen usf." (Popper 1977: 124) oder über irgendwelche makrosoziologischen Gesetze „sui generis“ zu erklären suche. Im Zentrum der situationslogischen Analysen der so verstandenen ,individualistischen“ Soziologie stünden gleichwohl aber vor allem die institutionellen Strukturen und die jeweiligen, oft unbeabsichtigten strukturbildenden Folgen des "situationsgerechten“ rationalen Handelns der Akteure, als makrosoziologische Gegebenheiten. Genau deshalb aber könne die Soziologie, etwa gegenüber einer „reduktionistischen“ Psychologie, eine eigene Fachidentität und Autonomie beanspruchen. Kurz: Das Konzept der Situationslogik und die Idee einer ,autonomen Soziologie“ folgen dem Prinzip des „Methodologischen Individualismus“, sind aber eben kein „Methodologischer Psychologismus".

Die Beziehungen des Konzeptes der Situationslogik zum Modell der soziologischen Erklärung und seinen drei grundlegenden Schritten liegen auf der Hand. Danach ist die erste Aufgabe der situationslogischen soziologischen Analyse die Rekonstruktion und modellierende Beschreibung der objektiven Situation der Akteure. Sie bedeutet bereits ein „Verstehen“ der Akteure - ohne sie selbst zu fragen! Denn dieses Verstehen ist nach Popper ein grundsätzlich objektives Verstehen, da es an den objektiv gegebenen Eigenschaften der Situation anknüpft. Mit dem Hinweis auf das objektive

${ }^{4}$ Vgl. Popper 1977: 112 ff. Vgl. auch Popper 1974: 115ff., 1974b, 1982, sowie die spätere Zusammenfassung bei Popper 1994 und die neueren Interpretationen des Konzepts bei Matzner und Jarvie 1998. 
Verstehen, man könnte auch sagen: auf die objektive Hermeneutik, bei der Situationsanalyse wird betont, da $B$ es nicht notwendig und nicht ratsam ist, bei der Beschreibung der Situation auf die Subjektivitäten und die individuellen Ideosynkrasien der Menschen einzugehen, sondern nur die „objektiven" Merkmale der Situation zu betrachten, die für das Handeln der Menschen unverrückbare Gegebenheiten auch dann sind, wenn sie das einstweilen nicht sehen oder nicht wahrhaben wollen. Alle geltenden sozialen Regeln - Normen, Verbote, rechtliche Bestimmungen - gehören dazu, ebenso wie die eher materiellen oder technischen Beschränkungen und Möglichkeiten des Handelns und die kulturellen Rahmungen und Strukturierungen der Definition der Situation. Die neueren Versionen des RC-Ansatzes berücksichtigen dies inzwischen mit dem Konzept der sozialen Produktionsfunktionen und der daran anschließenden Vorgänge des Framings. Und ohne weiteres können natürlich auch beobachtende Öffentlichkeiten dazu gehören.

Der zweite Teil des Argumentes von der objektiven Kraft der Situationslogik bezieht sich bei Popper auf die von ihm angenommene Selektionsregel eines "situationsgerechten" Handelns (Popper 1982: 120). Die Logik des situationsgerechten Handelns ist für Karl R. Popper die ,rationale“ Ausführung der Vorgaben der Logik der Situation. Dies sei aber, so Popper, keine "psychologische“ Gesetzmäßigkeit und keine empirische Eigenschaft des menschlichen Organismus, sondern eine Vorgabe, die in das Konzept der "Logik“ der Situation selbst einbeschlossen wäre. Karl R. Popper meint, daß dieser Teil der Situationslogik sogar derart selbstverständlich und so trivial sei, daß man ihn getrost vernachlässigen könne. Und das ist auch nicht unverständlich: Alles, was „rational“ ist, ist „logisch", und alles was „logisch" ist, ist ja in gewisser Weise tatsächlich selbstverständlich, a priori einsehbar und daher trivial und eigentlich nicht weiter erwähnenswert - wenngleich für „Logik" der betreffenden Erklärung unentbehrlich. Der einzige Unterschied zu den neueren Versionen der RC-Theorie ist die, daß dort die Logik der Selektion als eine - nicht immer nur: "triviale“ empirische Gesetzmäßigkeit des Handelns aufgefaßt wird, etwa in der Form der WE-Theorie.

Karl R. Popper nennt daher bekanntlich ein drittes Element der Situationslogik, von dem her gesehen es geradezu absurd erscheinen muß, bei der Erklärung der sozialen Prozesse alleine auf die psychischen Eigenschaften und insbesondere die Absichten und Motive der menschlichen Akteure Bezug zu nehmen: Die sozialen Prozesse und gesellschaftlichen Institutionen sind so gut wie nie das unmittelbare Ergebnis bewußter Planung, sondern „... in der Regel das indirekte, unbeabsichtigte und oft unerwünschte Nebenprodukt solcher Handlungen." (Popper 1977: 118; Hervorhebung nicht im Original) Es ist also die unentrinnbare Eigenlogik der unintendierten Folgen, die in der Tat dafür sorgt, daß die Menschen immer wieder von den Folgen ihres Tuns überrascht werden und daß sich die sozialen Prozesse oft genug auch gegen die Absichten, Wünsche und Bedürfnisse der Menschen nachhaltig durchsetzen. Gerade das Studium ,der unbeabsichtigten Rückwirkungen unserer Pläne und Handlungen“ (Popper 1977: 119) sei die wohl wichtigste Aufgabe der Soziologie.

Zur Erklärung der unentrinnbaren Eigendynamik sozialer Prozesse nimmt Karl R. Popper dann an, $\mathrm{daB}$ sich die drei Elemente - die Objektivität der Situation, die trivial-apriorische Regel des rationalsituationsgerechten Handelns und die Eigendynamik der unintendierten Folgen - zu einer festgefügten Einheit einer übergreifenden sozialen Logik zusammenschließen ließen. Die „Logik“ bei der Popperschen Situationslogik besteht, wie man leicht sieht, also aus just den drei Schritten des Modells der soziologischen Erklärung, jeweils verkoppelt zu mehr oder weniger langen ,interaktiven“ Sequenzen: Die „Logik der Situation“, formuliert in den Brückenhypothesen, die „Logik der Selektion“ in der WE-Entscheidungstheorie und die „Logik der Aggregation" in der Ableitung der strukturellen Folgen und der Erzeugung neuer struktureller Situationen und-eventuell-ganzer Sequenzen von Interaktionsdynamiken, oft ganz gegen die Absichten und Pläne der in den momentanen Situationen durchaus "rational" und daher verstehbar handelnden Menschen. Keines dieser drei Elemente darf fehlen, damit die Situationslogik ihre übergreifende „Logik" behält, auch das handlungstheoretische der WE-Theorie natürlich nicht, weil es sonst eine logische Lücke in der Situationslogik gibt.

In manchen Interpretationen der Popperschen Texte zur Situationslogik wird sogar gemeint, daß erst die neueren Entwicklungen des RC-Ansatzes, etwa bei James S. Coleman, zur vollen Entfaltung des Konzeptes vordrängen und daß Popper verschiedene Aspekte, die dort wesentlich stärker beachtet würden, vernachlässigt, wenngleich auch nicht gänzlich ubersehen habe, wie etwa Vorgänge der sozialen Interaktion (was wohl zutrifft), soziale Institutionen (was, wie wir gesehen haben, sicher nicht stimmt), die unintendierten Effekte und das Problem der Transformation individueller in kollektive Effekte (was ganz sicher nicht richtig ist) oder gar die „Interessen“ der Menschen (vgl.'Hedström et al. 1998: 351ff.). 
Wie immer die Rekonstruktion der Popperschen Texte aussieht: Die Einheit der drei Elemente des Modells der soziologischen Erklärung macht das Konzept der Situationslogik aus (vgl. dazu dann auch wieder: Hedström et al. 1998: 357 f.). Ihre Rekonstruktion für die beobachtbaren sozialen Prozesse ist die spezifische Aufgabe der Soziologie. Einige der wohl bedeutendsten Vertreter der Soziologie, wie etwa Alexis de Tocqueville, Karl Marx, Max Weber, wenn man nur etwas genauer hinsieht: auch Emile Durkheim, William I. Thomas, insbesondere aber Robert K. Merton, bestimmt auch Erving Goffman, Norbert Elias, Pierre Bourdieu, natürlich Raymond Boudon und James S. Coleman und durchaus auch, von seinem faktischen Vorgehen her wenigstens, Peter M. Blau, jedoch sicher nicht: Talcott Parsons und seine verschiedenen „system“-wie „handlungstheoretischen" Nachfolger und Nachahmer, haben diese Methode der soziologischen Situationslogik virtuos beherrscht und angewandt, gewiß auch ohne daß sie damit die Vorstellung oder gar die Bezeichnung „Rational Choice“ verbunden hätten. Es ist die soziologische Methode immer gewesen. Die mehr oder weniger großen Zwerge der neueren Versionen der Situationslogik im RC-Ansatz arbeiten dabei lediglich mit einigen aktualisierten, systematisierten und auch präzisierten theoretischen und methodischen Mitteln, die den klassischen Riesen der soziologischen Situationslogik einfach noch nicht zur Verfügung stehen konnten.

\section{Die „Alternative“ des Situationalismus}

Dieser Art der situationslogischen Erklärung der Entstehung und der Mobilisierung ethnischer Konflikte und anderer sozialer Prozesse treten Klaus Eder und Oliver Schmidtke in ihrem Beitrag in zwei Kritikpunkten und einem Alternativvorschlag, dem von den Autoren so genannten "Situationalismus“, entgegen (vgl. Eder/Schmidtke 1998).

\section{Die Kritik}

Der erste Kritikpunkt bezieht sich darauf, daß der RC-Ansatz grundsätzlich nicht in der Lage sei, die interaktive Konstitution kultureller Orientierungen zu erfassen (Eder/Schmidtke 1998: 424 f.). Die Situationsdefinitionen würden nur als ,äußere Randbedingung" angesehen, nicht aber in ihrer interaktiven Entstehung erklärt werden können. Das sei eine unvermeidliche Folge des solipsistisch-autonom gedachten Individuums, „, das seine 'rationale Wahl' nur vor oder nach der Interaktion mit anderen vornimmt.“ In Wahrheit unterlägen aber die Präferenzen, Motive und Interessen einer ,dauernden Formung und Veränderung in Interaktionssituationen", aus denen sich das Problem der Unsicherheit ergebe, das dem RC-Ansatz zwingend "aus dem theoretischen Blick“ gerate und dem nur mit einer "interaktionistischen“ Sichtweise beizukommen wäre.

Im zweiten Kritikpunkt (Eder/Schmidtke 1998: $425 \mathrm{ff}$.) wird dem RC-Ansatz vorgehalten, ,daß die Struktur und Dynamik von Handlungen und Handlungsketten erzeugenden Situationen nicht in den subjektiven Definitionen der Situation aufgehen. Es bleibt immer ein 'objektiver Rest'." Dieser Rest bestehe in zwei Sachverhalten. Erstens würden die Menschen „nicht zwischen gleichberechtigt nebeneinanderstehenden, mit vergleichbaren Durchsetzungschancen ausgestatteten 'Definitionen einer Situation “" ,'wählen'“, sondern sie „wählen das, was sich in der Interaktion - oft gegen den Willen und ihre Handlungspläne - als das heraus stellt, was den Machtverhältnissen entspricht." Und was dann geschieht, folge zweitens eben nicht den Absichten der Menschen, sondern „überrascht die Akteure selber. Akteure versuchen immer nur das Beste aus der Situation zu machen, sich in ihr so rational wie möglich zu verhalten (wobei sie wieder neue Überraschungen produzieren).“ Und wie "hochkompetent auch Akteure sein mögen, die Koordination ihrer Handlungen und die damit verbundenen Effekte auf die Reproduktion eines Handlungszusammenhangs geschehen ohne ihr Zutun." Deshalb werde beim RC-Ansatz das ,'Strukturelle' einer Situation (deren Entzifferung die Aufgabe der Soziologie ist) ... erst gar nicht zum Thema." Vielmehr müsse man, um dieses grundlegende Defizit des RC-Ansatzes zu beheben, ,über die Situation in den Köpfen der Beteiligten hinausgehen und die Situation, auf die sich die Beteiligten eingelassen haben, also die Situation außerhalb des Kopfes, genauer bestimmen".

Die kritisierten Punkte lassen sich in knapper Weise dann wohl so zusammenfassen: Dem RCAnsatz gelinge es schon von seiner Grundkonstruktion her nicht, die interaktive Konstitution von kulturellen Bezugsrahmen oder anderen strukturellen Gegebenheiten zu erfassen und er vernachlässige systematisch die objektiven Strukturen, seien es die objektiven Verhältnisse in der Situation oder seien es die strukturbildenden Eigendynamiken, die sich hinter dem Rücken der Akteure und gegen ihre Absichten einstellten. 


\section{Die Alternative}

Die vorgeschlagene Alternative des „Situationalismus" zielt dann allgemein genau auf die Überwindung dieser vermeintlichen Defizite ab (Eder/ Schmidtke 1998: 427): Es solle nun - anders als das angeblich beim RC-Ansatz schon von dessen Grundannahmen her möglich sei - endlich die der Soziologie "geschuldete Annahme“ der objektiven Strukturiertheit der Situationen und insbesondere die sich "'hinter dem Rücken von Akteuren'“ und ,'zwischen den Köpfen von Akteuren'“ einstellende „Koordination von Handlungsereignissen unabhängig von den Motiven der Akteure“ berücksichtigt werden.

Dazu werden zwei spezielle Vorschläge zur „Objektivierung" von Situationen gemacht (Eder/ Schmidtke 1998: 428ff.): Situationen seien erstens durch Normen und zweitens durch „dritte“ Akteure, durch Beobachter des Geschehens, wie etwa eine Öffentlichkeit oder ein Publikum, objektiv strukturiert. Daraus ergäben sich allerlei Folgen für eine angemessenere Analyse ethnischer Konflikte (Eder/Schmidtke 1998: $431 \mathrm{ff}$.). Insbesondere sei nun ein systematischer Zugang zu den auch medial vermittelten ,identitären Praktiken“ ethnischer Unternehmer möglich, ,in denen kulturelle Differenzen zwischen sozialen Gruppen legitimiert werden und Identitäten hergestellt werden" und über die versucht werde, bestimmte „Interpretationen sozialer Realität“ in einer beobachtenden Öffentlichkeit durchzusetzen. Erst mit diesem ,alternativen situationstheoretischen Modell“", das, ganz anders als der RC-Ansatz, „relational und strukturorientiert“" sei, könne das gelingen, was eigentlich der Kern der soziologischen Analyse und das grundlegende Problem jedes RC-Ansatzes sei: „daß nämlich kollektives Handeln und die Dynamik ethnischer Differenzierung sich nicht aus rationalen Entscheidungen interagierender Akteure ergeben, sondern aus der Logik sozialer Interaktionsstrukturen, aus Situationslogiken $\mathrm{zu}$ verstehen und $\mathrm{zu}$ erklären sind.“

\section{Jenseits wovon?}

Wenn die von Eder und Schmidtke vorgetragene Kritik am RC-Ansatz zuträfe, dann wäre in der Tat zu fragen, was er, nicht nur für die Erklärung ethnischer Konflikte, eigentlich wert sei und ob es nicht tatsächlich besser wäre, weiterhin ,jenseits von 'Rational Choice" in einem der - in der Konkurrenz etwa zur Ökonomie bislang ja nicht allzu erfolgreichen - herkömmlichen soziologischen $\mathrm{Pa}$ radigmen zu verbleiben. Wir gehen die Punkte der Reihe nach durch.

\section{Soziale Konstitution}

Die „einfachen“ Versionen des RC-Ansatzes gingen (und gehen) von dem neoklassischen Modell des homo oeconomicus aus. Deshalb waren in der Tat auch alle die Einwände nicht unberechtigt, die auf die eigenständige Wirkung von Normen, Werten, Identitäten und dergleichen sowie auf die sehr begrenzte Rationalität des Handelns verwiesen. Diese Begrenzungen für die „rationale“ Erklärung auch des normativen oder des wertgebundenen Handelns sind mit der Vervollständigung des RCAnsatzes um das Konzept des Framings inzwischen beseitigt. Das können Eder und Schmidtke nicht dementieren. Kritisiert wird jedoch weiter, daß die Frames in dem Ansatz nur als gegebene Randbedingungen angenommen werden könnten, während die Genese ihrer sozialen „Konstitution“ grundsätzlich nicht zu erfassen sei, weil die Entstehung und die Änderung der Erwartungen und Bewertungen der Akteure nicht erklärt werden könne.

Das ist ein geläufiger Vorwurf, der die Entwicklung und Fortführung des Ansatzes von Beginn an begleitet hat (vgl. u.a. Trapp 1886; Srubar 1992, 1994; Burkart 1994; Egger/di Campo 1997; Dallinger 1998). Es ist die Spezialversion eines noch älteren Einwandes, wonach das RC-Modell der soziologischen Erklärung nur das „isolierte“ und „punktuelle“ Handeln einzelner Menschen erklären könne, wohingegen die Erklärung der Genese sozialer Strukturen und deren interaktive Konstitution, auch die von Normen oder kulturellen Codierungen, Vorgänge seien, die sich dem Modell der soziologischen Erklärung, zumal dann, wenn es die WE-Theorie benutze, grundsätzlich verschließen würde.

Diese Auffassungen sind nach allen, sozusagen: „,immer schon“ verfügbaren Darstellungen der Arbeitsweise und Argumentation des RC-Ansatzes, gelinde gesagt, unbegreiflich und selbst offenbar ein Fall des sozialen Framings, der kollektiven Konstruktion von Überzeugungssystemen: Nicht nur sind die Entstehung und der Wandel etwa von Institutionen, dem wohl wichtigsten Fall der Entstehung gesellschaftlicher Strukturen und der Bestimmung von Erwartungen und Bewertungen, und insbesondere Revolutionen und soziale Bewegungen das Thema des RC-Ansatzes von Anfang 
an gewesen. Der RC-Ansatz hat gerade hier Ergebnisse vorgelegt, die unter der Hand inzwischen von den Konkurrenzparadigmen übernommen, teilweise als deren Leistungen ausgegeben werden - wie etwa die Auflösung des bereits erwähnten Tocqueville-Paradox, wonach Revolutionen gerade erst dann zu entstehen pflegen, wenn die größte Deprivation schon beseitigt ist, weil dann die Erfolgserwartungen von null auf größer als null steigen.

Es gibt ferner auch keinerlei Grund für die Behauptung, daß die interaktive Konstitution von $\mathrm{Co}$ dierungen, der Entstehung und Wirkung von „Werten" und „Moral“, von ethnischen Grenzziehungen oder religiösen Glaubenssystemen, mit dem Ansatz grundsätzlich nicht zu bearbeiten seien. Dabei wird durchaus nicht (nur) an spieltheoretische Modellierungen solcher Interaktionen gedacht, obwohl auch das schon für manche Vorgänge ausgesprochen weiterführend ist, wie etwa die Modellierung des Wettlaufs um die Bemächtigung der Machtmittel zur Durchsetzung einer bestimmten Verfassung. Eine Möglichkeit der Erklärung des Vorgangs des sozialen Framings als Grundlage der Erklärung der sozialen Konstitution von geteilten Situationsdefinitionen wurde oben in dem Beispiel über ethnische Konflikte ausführlich skizziert. Dazu muß, es sei wiederholt, nur die Annahme gemacht werden, daß die Akteure nicht bloß „isoliert" handeln, sondern sich und ihr Tun auch beobachten, wahrnehmen und aufgrund dieser Wahrnehmungen, freilich nach angebbaren Gesetzen, ihre „Definition“ der Situation ändern. Das alles ist ohne weiteres im Rahmen der Mittel des RC-Ansatzes, spätestens mit seiner, wie es heißt, „kognitivistischen“ Erweiterung um das FramingKonzept, möglich. Herkömmlichere Versionen des RC-Ansatzes benutzen hier das Konzept des „signalling“ bzw. das des Bayesianischen Lernens (vgl. etwa Rasmusen 1991: 210 ff.; Breen/Garcia-Penalosa 1996). Das Framing-Konzept integriert darüberhinaus auch die Orientierung an kulturellen Modellen und „Einstellungen“ und gibt dabei die genauen theoretischen Bedingungen und Gesetzmäßigkeiten an, unter welchen Umständen sich Frames und deren Codierungen - aufgrund natürlich auch von Beobachtungen, Wahrnehmungen und der „Interpretation“ von Symbolen - ändern, sich verfestigen oder sich abschwächen oder auch so bleiben wie sie sind. Es ist eine $R C$-Erklärung für die Bedingungen und die Folgen, unter denen sich Menschen ,rational“ oder ,irrational“" verhalten, etwa unter low-cost-Umständen oder bei der „unbedingten“ Geltung eines „Wertes“.
Das Modell der soziologischen Erklärung läßt sich, anders als die Kritik annimmt, auch vollkommen problemlos auf ganze Ketten interaktiver Sequenzen solcher Vorgänge, natürlich auch auf solche des "Aushandelns“, natürlich auch unter Einschluß dritter beobachtender Parteien, natürlich auch in strategischer Absicht zur Beeinflussung einer Öffentlichkeit oder in der Befolgung von Normen anwenden. Auch daher sind die Vorhaltungen schon einigermaßen unerfindlich, ebenso wie die Behauptung, der RC-Ansatz habe es zwingend mit isolierten, autonomen, solipsistischen Akteuren zu tun. Die diversen Markt- und Tauschmodelle, auch schon die des neoklassischen RC-Ansatzes, insbesondere aber die Präzisierungen dessen, was in der interaktionistischen Soziologie etwas locker mit „Aushandeln“ bezeichnet wird, in den verschiedenen Verhandlungsmodellen und Koalitionstheorien des RC-Ansatzes, befassen sich ebenfalls mit der „interaktiven Konstitution" und mit dem „Kampf“ um die Durchsetzung gewisser Interessen, gerade auch in Situationen, die von Macht und struktureller Ungleichheit durchzogen sind. Und nirgendwo ist festgelegt, daß sich der Gegenstand des interaktiv-strategischen Geschehens nur auf die Transaktionen materieller Güter oder institutioneller Rechte und nicht auch auf die Durchsetzung und das „Aushandeln“ von kulturellen Situationsdefinitionen als Ressourcen beziehen könnte (vgl. dazu auch die interaktionistisch-strategische Reformulierung des Kulturkonzepts bei Swidler 1986 oder bei Wimmer 1996).

In die Behauptung, der RC-Ansatz könne die interaktive soziale Konstitution von kulturellen Rahmungen nicht erklären, wird gelegentlich ein Argument eingeflochten, dessen Ernsthaftigkeit aus den Texten nicht leicht zu erschließen ist. Es wird inauguriert, daß sich die interaktive Konstitution nicht über kausale Sequenzen von Wirkungen modellieren lasse, weil sich das interaktive Geschehen und die Änderungen der Zustände der Akteure sozusagen simultan ereigneten, eben , in der Interaktion mit anderen" (Eder/Schmidtke 1988: 424), und nicht, wie es das Konzept der Sequenz nahelegt, durch ein zeitbezogenes Wechselspiel von Akten, Beobachtungen, Wirkungen, erneuten Akten, Beobachtungen und Wirkungen usw. Das Argument von der grundsätzlichen Simultanität der interaktiven Konstitution und fortlaufenden Änderung der Präferenzen und Erwartungen kann eigentlich nicht ernst gemeint sein, denn sonst würde sich ja nichts „ändern“ und die sozialen Bewegungen würden sich nicht ,bewegen" können. Wenn aber Zeit vergeht, dann ist sie auch in Sequenzen der Änderung von Zuständen und Eigenschaften zerlegbar, und der Proze $\beta$ muß auch so modelliert werden. $\mathrm{Da} \beta$ das anders ginge, kann man nur angesichts verschwommener sprachlicher Andeutungen der „Gleichzeitigkeit" annehmen. Selbstverständlich sind empirisch die 
einzelnen Phasen von Akten, Beobachtungen und Wirkungen nicht immer eindeutig zu trennen, und es gibt wohl auch Interaktionssequenzen mit sehr kleinen Zeitabständen, etwa bei den Auseinandersetzungen zwischen gangs von Jugendlichen oder in hitzigen Diskussionen zwischen Wissenschaftlern. Aber alles das sind keine wirklich gravierenden theoretischen Einwände. Es sind eher MeB- und Operationalisierungsprobleme - oder solche der Infinitesimalrechnung, die man zur Sequenzierung unendlich kleiner Schritte erfunden hat. Jener spezielle Fall der tatsächlich ,simultanen“ Konstitution von gemeinsamen Situationsdefinitionen, der als Ko-Orientierung bekannt ist (vgl. dazu die immer noch lesenswerte Darstellung von Siegrist 1970), bildet ohnehin kein besonderes Erklärungsproblem: Das ist die gegenseitige gedankliche Einfühlung in den jeweils anderen in der punktuellen Situation - mit einem daran orientierten anschlieBenden Handeln, dessen beobachtbare Ergebnisse dann die (Ko-)Orientierungen freilich wieder verändern könnten.

Kurz: Im Rahmen des RC-Ansatzes bzw. im Modell der soziologischen Erklärung ist die Erklärung der interaktiven sozialen Konstitution von Strukturen ohne weiteres möglich, und mit dem Konzept des sozialen Framings gehört ohne Probleme auch die Erklärung der interaktiven sozialen Konstitution kultureller Bezugsrahmen und kollektiver Situationsdefinitionen dazu..$^{5}$ Allerdings macht der RC-Ansatz hier eine - durchaus falsifizierbare - deutliche Aussage: Alle nachhaltigen Versuche und Prozesse zur Durchsetzung bzw. Verbreitung der kulturellen Codierungen haben auch einen strukturellen Hintergrund: Die durch die Verteilung des spezifischen Kapitals strukturierten materiellen Interessen der Gruppen, vor denen die jeweiligen Codierungen in den Augen der Akteure erst ihren Sinn und ihre Legitimität finden. Insofern stellt der RC-Ansatz die Erklärung der ethnischen Konflikte in der Tat (wieder) vom Kopf auf die Füße und flüchtet sich nicht in die derzeit sehr in Mode gekommenen konstruktivistischen Spekulationen im Zusammenhang mit diesem Thema.

\section{Strukturen}

Damit ist auch schon die zweite Vorhaltung beantwortet, wonach der RC-Ansatz keine objektiven sozialen Strukturen kenne. Diese Behauptung ist noch unbegreiflicher. Selbstverständlich sind die Situationen, in denen menschliche Akteure handeln, objektiv strukturiert, sei es durch soziale Pro-

5 Vgl. dazu aus der expliziten Sicht des RC-Ansatzes: Esser 1993, Abschnitt 27.4; Esser 1996c, Abschnitt V; Esser 1999, Kapitel 25 und 51. duktionsfunktionen, durch materielle Opportunitäten, institutionelle Regeln, geteilte kulturelle Muster, soziale Beziehungen und Netzwerkstrukturen, sei es auch durch beobachtende Dritte (oder auch noch mehr, wie in einer Gruppe, die größer ist als eine Triade). Und ebenso selbstverständlich wird nicht davon ausgegangen, daß die Akteure irgendwie in einem macht- oder herrschaftsfreien Raum agieren, keine Beziehungen unterhielten und souverän ihre Absichten durchsetzen könnten. Auch stehen die Alternativen natürlich nicht alle ,gleichberechtigt nebeneinander, mit vergleichbaren Durchsetzungschancen", wiè Eder und Schmidtke schreiben. Gerade das wird ja in der Modellierung der EU-Gewichte für die Erklärung des Handelns systematisch berücksichtigt. „Rational Choice“ heißt nicht zwingend „Indifferenz" zwischen Alternativen, und die "Unsicherheit" in einer Situation ist auch nur ein Spezialfall der Handlungssituation, über den es gerade für die interaktionistische Soziologie beim RC-Ansatz und von der WE-Theorie viel zu lernen gibt.

Vollends unbegreiflich ist dann die Einlassung, der RC-Ansatz könne soziale Prozesse als eigendynamische Vorgänge der Strukturierung nicht behandeln. Schon bei der Darstellung der RC-Erklärung ethnischer Konflikte, spätestens bei der des Popperschen Konzepts der Situationslogik dürfte die Absurdität dieses Vorwurfes deutlich geworden sein. Der RC-Ansatz bzw. das Modell der soziologischen Erklärung waren von Beginn an ein Unternehmen, bei dem gerade die aggregierten, von den Motiven der Akteure unabhängigen eigendynamischen strukturellen Effekte im Mittelpunkt des Erklärungsinteresses und der vorgelegten Erklärungen standen. Das war schon bei Adam Smith mit seiner Metapher von der unsichtbaren Hand so gewesen, bei Robert K. Merton mit seiner Idee von den unbeabsichtigten Folgen des absichtsvollen Handelns bis hin zu - sagen wir - Raymond Boudon und den von ihm besonders gern und überzeugend analysierten paradoxen Effekten. Das systematische Auseinanderfallen der - kurzfristigen, situationsorientierten subjektiven - Rationalität des Handelns in einer Situation und mancherlei ungeplanter kollektiv irrationaler struktureller Folgen ist das gemeinsame Thema aller dieser Varianten des RC-Ansatzes immer gewesen. Die Analyse der interaktiven sozialen Konstitution von ethnischen Codierungen wäre nichts anderes als ein Anwendungsfall der dabei benutzten theoretischen Instrumente. Und gerade die neoklassische Ökonomie, als die wohl extremste Form des RC-Ansatzes, ist eben keine Handlungs- oder Ent- 
scheidungstheorie, sondern vor allem anderen eine Theorie der Erklärung emergenter Prozesse, vor allem von Märkten, von Konjunkturen oder von Pfaden des Wachstums oder des Niedergangs. Und sie hält am homo oeconomicus gerade deshalb fest, weil sich damit just diese Aggregationen noch am leichtesten modellieren lassen, um die es eigentlich nur geht.

\section{Situationalismus}

Damit finden wir uns in einer etwas seltsamen, aber nicht ungewohnten Situation wieder: Dem RC-Ansatz werden einige Möglichkeiten und Leistungen grundsätzlich abgesprochen, die von Beginn an zu seinen zentralen Bestandteilen, programmatischen Absichten und nachweisbaren Leistungen gehören. Die Vorhaltungen beruhen fast ausschlieBlich auf unbegründeten Zuschreibungen von grundlegenden „Grenzen" und Defiziten, deren Haltlosigkeit in einer inzwischen fast jahrzehntelangen Debatte und zu sehr verschiedenen Anlässen immer wieder detailliert begründet wurde. ${ }^{6}$ Als "Alternative“ wird das Konzept des "Situationalismus“ vorgeschlagen. Diese „Alternative“ ist aber, wenigstens in der vorliegenden Darlegung, im wesentlichen nichts anderes als das, was just der RC-Ansatz immerschon getan hat, zu dem es mit dem Konzept der Situationslogik von Karl R. Popper eine praktisch identische methodologische Formulierung gab und die selbst in Hinsicht auf den inhaltlichen Vorschlag des Einbezugs einer „beobachtenden“ Öffentlichkeit in das Geschehen von seiten des RC-Ansatzes inzwischen sogar auch schon empirisch bearbeitet wurde (vgl. Lüdemann 1998).

Eder und Schmidtke verwechseln - mit anderen, wie etwa Megely (1998) - offenbar den RC-Ansatz, das Modell der soziologischen Erklärung und die damit jeweils verbundene Position des „Methodologischen Individualismus“ mit dem längst überholten reduktionistischen Psychologismus und Atomismus, gegen den sich schon Popper gewandt hatte. Und sie bemerken, erneut wie andere, etwa Mozetic (1998), nicht, daß das Modell der soziologischen Erklärung und die Erweiterung der RC-Theorie um die Framingprozesse auch alle jene Vorbehalte gegenstandslos machen, die für die einfache Übernahme der neoklassischen Ökonomie in die Soziologie noch zugetroffen haben mögen.

\footnotetext{
${ }^{6} \mathrm{Vgl}$. die Ausführungen bei Vanberg (1975) oder bei Opp (1979) auch schon für wesentlich „reduktionistischere“ Varianten des RC-Ansatzes; vgl. auch die Auseinandersetzung mit der sog. Figurationssoziologie, die ähnliche Einwände gegen den ,Methodologischen Individualismus" glaubte formulieren zu müssen, bei Esser (1984).
}

Bei dem Vorschlag des „Situationalismus“ bleibt zudem gänzlich offen, wie die vorgetragenen Absichten denn theoretisch - über die vorgestellte Typologie hinaus - umgesetzt werden sollen, insbesondere über welche Art von „Logik“ sich die Logik des „Situationalismus“ denn eigendynamisch oder sonstwie bewegen soll, von der Eder und Schmidtke nur sagen, daß sie nicht deterministisch und nicht linear wäre.

\section{Wie weiter?}

Die Soziologie befindet sich derzeit in keiner besonders guten Verfassung. Vieles ist in Bewegung, und ob sich eine Richtung schließlich durchsetzt, ob es bei der multiplen Paradigmatase der gegenwärtigen Situation bleibt oder ob es vielleicht doch zur Einheit der Gesellschaftswissenschaften kommt, ist nicht auszumachen. Dabei spricht vieles für und kaum etwas gegen eine integrierende erklärende Perspektive mit einem eigenen Platz einer durchaus autonom bleibenden Soziologie darin, etwa als mit dem Modell der soziologischen Erklärung betriebene system- und evolutionstheoretisch orientierte Soziologie unter der Leitidee der strukturellen Selektion auf handlungstheoretischer Grundlage (vgl. dazu die Ausführungen bei Schmid 1998: 264ff.).

Bisher hat die Persistenz der verschiedenen Schulen eine durchaus wichtige Funktion gehabt: Es waren Anmahnungen auf die Berücksichtigung von Aspekten, ohne die eine soziologische Erklärung unvollständig bleiben müßte: Funktionale Gleichgewichte und soziale Systeme, Normen und Werte, Opportunitătsstrukturen und Cleavages, symbolische Interaktion und Sinn, Anreize und egoistischer „Rational Choice“ sind allesamt zentrale Bestandteile des sozialen Geschehens und müßten in soziologischen Erklärungen einen systematischen und gegenseitig aufeinander bezogenen Platz finden.

Das Modell der soziologischen Erklärung mit der „RC+“-Vervollständigung ist ein Vorschlag zur Zusammenführung aller dieser Elemente und zur Überwindung der Partikularparadigmen, die sich die Soziologie leistet, zugunsten einer integrativen und nicht-"reduktionistischen" erklärenden Sozialtheorie. Niemand würde bestreiten wollen, daß dieses Vorhaben, wie alle anderen, seine Grenzen hat. Und es wäre in der Tat wichtig, hilfreich und interessant, diese Grenzen, in auch lebhaften Kontroversen, ernsthaft auszuloten. Dazu müßten aber - endlich einmal - andere Einwände zu hören sein als - immer wieder - die, daß der RC(+)-Ansatz vom autonomen Individuum ausgehen und die Er- 
wartungen und Präferenzen als gegeben ansehen müsse, die institutionellen Umstände und die relationalen Verflechtungen ignoriere, die Genese von Strukturen und die Emergenz kollektiver Folgen nicht erklären könne, mit der interaktiven Konstitution von Situationsdefinitionen und mit Rückbezüglichkeiten und Reflexivitäten nicht zu Rande komme, keine Macht, keine Zwänge und keine Objektivitäten kenne und mit unintendierten Folgen und nicht-deterministischen oder nicht-linearen Eigendynamiken nichts anfangen könne oder gar, daß der Ansatz einer "soziologischen" Alternative bedürfe, deren Kern die, jetzt erst entdeckte, Idee der Situationslogik wäre.

Selbst gegen den in der Tat noch recht „reduktionistischen" verhaltenstheoretischen Ansatz waren die meisten dieser Einwände schon unangebracht. Und das sind sie erst recht gegen die neueren RC(+)-Ansätze mit ihrem systematischen Einbezug auch von kognitiven und symbolisch-interaktiv erzeugten kulturellen Prozessen. Es bleibt dann, wie schon früher, die Frage, warum insbesondere die so lange schon ausgeräumten Einwände der Strukturlosigkeit und des psychologistischen Subjektivismus des $\mathrm{RC}(+)$-Ansatzes gleichwohl immer wieder neu hervorgeholt und publiziert werden - und warum dagegen Alternativen gutgeheißen werden, die entweder keine sind oder die für eine bessere Lösung nur dann gehalten werden können, wenn man die Voraussetzungen einer angemessenen (soziologischen) Erklärung nicht kennt oder nicht ernstnimmt. Stereotype Vorhaltungen erzeugen natürlich auch gerne stereotype Reaktionen. Die schon bei den fritheren Anlässen immer etwas ratlose Erklärung für die stete Wiederholung der üblichen soziologistischen Einwände und Grenzziehungen gegen die Bemühungen zu einer integrativen Sozialtheorie hatte eine gewisse Ähnlichkeit mit der (RC-)Erklärung der ethnischen Konflikte. Es war die Vermutung eines zwar wissenschaftspolitisch verständlichen, argumentativ aber kaum begründbaren Revierverhaltens der um ihre Eigenständigkeit und um ihr spezifisches Kapital besorgten Paradigmen der herkömmlichen Soziologie. Das war sicher eine einfache Antwort. Aber deshalb alleine muß sie ja nicht falsch sein. Immer noch nicht.

\section{Literatur}

Axelrod, R., 1987: Die Evolution der Kooperation, München: Oldenbourg

Banton, M., 1967: Race Relations, London: Tavistock Publ.
Banton, M., 1993: Racial and Ethnic Competition, Cambridge

Banton, M., 1994: Modeling Ethnic and National Relations. Ethnic and Racial Studies 17: 1-17

Bergmann, W., 1998: Pogrome: Eine spezifische Form kollektiver Gewalt. Kölner Zeitschrift für Soziologie und Sozialpsychologie 50: 644-665

Breen, R./Garcia-Penalosa, C., 1997: A Rational Learning Model of Gender Segregation in Labor Markets. Economics Discussion Paper N. 135, Oxford

Brubaker, R./Laitin, D.D., 1998: Ethnic and National Violence. Annual Review of Sociology 24: 423-452

Burkart, G., 1994: Die Entscheidung zur Elternschaft: Eine empirische Kritik von Individualisierungs- und Rational-Choice-Theorien, Stuttgart: Enke

Calic, M.-J., 1996: Krieg und Frieden in Bosnien-Hercegovina, 2. erweiterte Aufl., Frankfurt/M.: Suhrkamp

Dallinger, U., 1998: Der Konflikt zwischen familiärer Pflege und Beruf als handlungstheoretisches Problem. Zeitschrift für Soziologie 27: 94-112

Diekmann, A./Preisendörfer, P., 1998: Umweltbewußtsein und Umweltverhalten in Low- und High-Cost-Situationen: Eine empirische Überprüfung der Low-Cost-Hypothese. Zeitschrift für Soziologie 27: 438-453

Druwe, U./Kunz, V. (Hrsg.), 1998: Anomalien in der Handlungs- und Entscheidungstheorie, Opladen: Leske+Budrich

Eder, K./Schmidtke, O., 1998: Ethnische Mobilisierung und die Logik von Identitätskämpfen: Eine situationstheoretische Perspektive jenseits von „Rational Choice“. Zeitschrift für Soziologie 27: 418-437

Egger, M./di Campo, A., 1997: Was Sie schon immer über das Verhalten in sinkenden U-Booten wissen wollten: Eine Replik zu Hartmut Essers Aufsatz „Die Definition der Situation". Kölner Zeitschrift für Soziologie und Sozialpsychologie 49: 306-317

Esser, H., 1980: Aspekte der Wanderungssoziologie, Darmstadt/Neuwied: Luchterhand

Esser, H., 1984: Figurationssoziologie und Methodologischer Individualismus: Zur Methodologie des Ansatzes von Norbert Elias. Kölner Zeitschrift für Soziologie und Sozialpsychologie 36: 667-702

Esser, H., 1985: Soziale Differenzierung als ungeplante Folge absichtsvollen Handelns: Der Fall der ethnischen Segmentation. Zeitschrift für Soziologie 14: 435-449

Esser, H., 1988: Ethnische Differenzierung und moderne Gesellschaft. Zeitschrift für Soziologie 17: 235-248

Esser, H., 1996: Soziologie: Allgemeine Grundlagen, (2. Aufl.), Frankfurt a.M./New York: Campus

Esser, H., 1996a: Die Mobilisierung ethnischer Konflikte. S. 63-87 in: K.J. Bade (Hrsg.), Migration-EthnizitätKonflikt, IMIS-Schriften 1, Osnabrück: Universitätsverlag Rasch

Esser, H., 1996b: Ethnische Konflikte als Auseinandersetzung um den Wert von kulturellem Kapital. S. 64-99 in: W. Heitmeyer/R. Dollase (Hrsg.), Die bedrängte Toleranz: Ethnisch-kulturelle Konflikte, religiöse Differenzen und die Gefahren politisierter Gewalt, Frankfurt/ M.: Suhrkamp 
Esser, H., 1996c: Die Definition der Situation. Kölner Zeitschrift für Soziologie und Sozialpsychologie 48:134

Esser, H., 1997: Die Entstehung ethnischer Konflikte. S. 876-894 in: Stefan Hradil (Hrsg.), Differenz und Integration. Verhandlungen des 28.Kongresses der DGS, Frankfurt M./New York: Campus

Esser, H., 1999 (im Erscheinen): Soziologie: Spezielle Grundlagen, Frankfurt a.M./New York: Campus

Fearon, J.D./Laitin, D.D., 1996: Explaining Interethnic Cooperation. American Political Science Review 90: 715-735

Ganter, S., 1995: Ethnizität und ethnische Konflikte: Konzepte und theoretische Ansätze für eine vergleichende Analyse, Freiburg: ABI, Arnold-Bergstraesser-Institut Gartner, S.S./Segura, G.M., 1997: Appearances Can Be Deceptive: Self-Selection, Social Group Identification, and Political Mobilization. Rationality and Society 9: 131-161

Giegel, H.-J., 1998: Gesellschaftstheorie und Konfliktsoziologie. S. 9-28 in: H.-J. Giegel (Hrsg.), Konflikt in modernen Gesellschaften, Frankfurt/M.: Suhrkamp

Granovetter, M., 1978: Threshold Models of Collective Behavior. American Journal of Sociology 83: 14201443

Hardin, R., 1995: One For All: The Logic of Group Conflict, Princeton, N.J.: Princeton University Press

Hechter, M., 1995: Explaining National Violence. Nations and Nationalism 1: 53-68

Hechter, M./Friedman, D./Appelbaum, M., 1982: A Theory of Ethnic Collective Action. International Migration Review 16: 412-434

Hedström, P./Swedberg, R./Udéhn, L., 1998: Popper's Situational Analysis and Contemporary Sociology. S. 339365 in: E. Matzner/I.C. Jarvie (Hrsg.), Papers from the Vienna Workshop on Popper's Situational Analysis and the Social Sciences, Part I, Band 28 der Philosophy of the Social Sciences (Special Issue), Thousand OaksLondon-New Delhi,

Horowitz, D.L., 1985: Ethnic Groups in Conflict, Berkeley, Cal.: University of California Press

Laitin, D.D., 1995: National Revivals and Violence. Archives Europeénnes de Sociologie 36: 3-43

Lohmann, S., 1994: The Dynamics of Informational Cascades: The Monday Demonstrations in Leipzig, East Germany, 1989-1991. World Politics 47: 42-101

Lüdemann, Chr., 1998: Das Mikro-Makro-Modell von James S. Coleman: Kontrollübertragungen und fremdenfeindliche Gewalt. S.157-179 in: H.-P. Müller/M. Schmid (Hrsg.), Norm, Herrschaft und Vertrauen: Beiträge zu James Colemans Grundlegung der Sozialtheorie, Opladen: Westdeutscher

Matzner, E./Jarvie, I.C. (Hrsg.), 1998: Papers from the Vienna Workshop on Popper's Situational Analysis and the Social Sciences, Part I, Band 28 der Philosophy of the Social Sciences (Special Issue), Thousand OaksLondon-New Delhi

Meleghy, T., 1998: Verhaltenstheorie und Handlungstheorie: Versuch einer Abgrenzung. S. 227-262 in: A. Ba$\log /$ M. Gabriel (Hrsg.), Soziologische Handlungstheo- rie: Einheit oder Vielfalt, Sonderband 4 der Österreichischen Zeitschrift für Soziologie, Opladen: Westdeutscher

Mozetic, G., 1998: Wieviel muß die Soziologie über Handlungen wissen? Eine Auseinandersetzung mit der Rational-Choice-Theorie. S. 199-226 in: A. Balog/M. Gabriel (Hrsg.), Soziologische Handlungstheorie: Einheit oder Vielfalt, Sonderband 4 der Österreichischen Zeitschrift für Soziologie, Opladen: Westdeutscher

Oliver, P./Marwell, G./Texeira, R., 1985: A Theory of the Critical Mass I: Interdependence, Group Heterogeneity, and the Production of Collective Action. American Journal of Sociology 91: 522-556

Opp, K.-D., 1979: Individualistische Sozialwissenschaft: Arbeitsweise und Probleme individualistisch und kollektivistisch orientierter Sozialwissenschaften, Stuttgart: Enke

Popper, K.R., 1974a: Das Elend des Historizismus, 4. Aufl., Tübingen: Mohr

Popper, K.R., 1974b: Towards a Rational Theory of Tradition. S. 120-135 in: K.R. Popper, Conjectures and Refutations: The Growth of Scientific Knowledge, 5. Aufl., London: Routledge and Kegan Paul

Popper, K.R., 1977 (zuerst 1945): Die offene Gesellschaft und ihre Feinde: Band 2: Falsche Propheten: Hegel, Marx und die Folgen, 5. Aufl, München: Mohr

Popper, K.R., 1982: Die Logik der Sozialwissenschaften. S: $103-123$ in: H. Albert/Th.W. Adorno/R. Dahrendorf/J. Habermas/H. Pilot/K.R. Popper: Der Positivismusstreit in der deutschen Soziologie, 10. Aufl., Darmstadt/Neuwied: Luchterhand

Popper, K.R., 1994: Models, Instruments, and Truth: The Status of the Rationality Principle in the Social Sciences. in: Karl R. Popper, The Myth of the Framework, London: Routledge

Rasmusen, E., 1991: Games and Information: An Introduction to Game Theory, Oxford/Cambridge, Mass.: Blackwell

Rucht, D., 1994: Modernisierung und neue soziale Bewegungen: Deutschland, Frankreich und USA im Vergleich, Frankfurt a.M./New York: Campus

Schelling, Th.C., 1978: Micromotives and Macrobehavior, New York/London: Norton

Schmid, M., 1998: Soziales Handeln und strukturelle Selektion: Beiträge zur Theorie sozialer Systeme, Opladen/Wiesbaden: Westdeutscher

Siegrist, J., 1970: Das Consensus-Modell: Studien zur Interaktionstheorie und zur kognitiven Sozialisation, Stuttgart: Enke

Srubar, I., 1992: Grenzen des „Rational-Choice“-Ansatzes. Zeitschrift für Soziologie 21: 157-165

Srubar, I., 1994: Die (neo-)utilitaristische Konstruktion der Wirklichkeit. Soziologische Revue 17: 115-121.

Stocké, V., 1998: Framing oder Informationsknappheit? Zur Erklärung der Formulierungseffekte beim AsianDisease-Problem. S. 197-218 in: U. Druwe/V. Kunz (Hrsg.), Anomalien in der Handlungs- und Entscheidungstheorie, Opladen; Leske+Budrich

Swidler, A., 1986: Culture in Action: Symbols and Strategies. American Sociological Review 51: 273-286 
Tajfel, H./Turner, J.C., 1986: Social Identity Theory of Intergroup Behavior. S. 7-25 in: S. Worchel/W. Austin (Hrsg.), Psychology of Intergroup Relations, 2. Aufl., Chicago: Nelson Hall

Taylor, M., 1987: The Possibility of Cooperation, Cambridge: Cambridge University Press

Trapp, M., 1986: Utilitaristische Konzepte in der Soziologie: Eine soziologische Kritik von Homans bis zur Neuen Politischen Ökonomie. Zeitschrift für Soziologie 15 : $324-340$

Vanberg, V., 1975: Die zwei Soziologien: Individualismus und Kollektivismus in der Sozialtheorie, Tübingen: Mohr
Weingast, B., 1998: Constructing Trust: The Politics and Economics of Ethnic and Regional Conflict. S. 163-200 in: V. Haufler, K. Soltan und E. Uslaner, Institutions and Social Order, Ann Arbor: University of Michigan Press

Wimmer, A., 1995: Interethnische Konflikte: Ein Beitrag zur Integration aktueller Forschungsansätze. Kölner Zeitschrift für Soziologie und Sozialpsychologie 47: 464-493

Wimmer, A., 1996: Kultur: Zur Reformulierung eines sozialanthropologischen Grundbegriffs. Kölner Zeitschrift für Soziologie und Sozialpsychologie 48: 401425 